\title{
POSTULATES FOR ABELIAN GROUPS AND FIELDS IN TERMS OF NON-ASSOCIATIVE OPERATIONS*
}

\author{
BY \\ B. A. BERNSTEIN
}

1. Object. The object of this paper is to present sets of postulates for abelian groups and fields in terms of the non-associative (and non-commutative) operations " -" and "/", the inverses of "+" and " $X$ " in a field. The postulates will thus treat directly the properties of the inverse operations in a field, properties important from the standpoint of operations in general, but perhaps not sufficiently emphasized in the usual treatment of groups and fields. Three sets of postulates will be given for fields. In each set, the postulates free from "/", taken by themselves, will form a set of postulates for abelian groups. Unlike other sets of postulates for fields known to me, the sets offered contain no (unconditioned) existence proposition other than one demanding that the class contain at least two elements. The consistency, necessariness, and sufficiency of the postulates are established by the usual methods. The postulates will be found to be simple and "natural".

2. Postulates (F) for fields. A class $K$ of elements will be a (non-trivial) field with respect to a pair of binary operations "-", "/" if $K,-$, / satisfy the postulates $\mathrm{N}, \mathrm{S}_{1}-\mathrm{S}_{3}, \mathrm{D}_{1}-\mathrm{D}_{6}$ following:

N. $K$ contains at least two distinct elements.

$\mathrm{S}_{1}$.

$$
a=a-(b-b)
$$

if $a, b$, and the combinations indicated are in $K$.

$\mathrm{S}_{2}$.

$$
a-(b-c)=c-(b-a),
$$

if $a, b, c$, and the combinations indicated are in $K$.

$\mathrm{S}_{3}$. If $a, b$ are in $K$ then $a-b$ is in $K$.

$\mathrm{D}_{1}$.

$$
a=a /(b / b)
$$

if $a, b$, and the combinations indicated are in $K$.

$\mathrm{D}_{2}$.

$$
a /(b / c)=c /(b / a)
$$

if $a, b, c$, and the combinations indicated are in $K$.

* Presented to the Society, April 11, 1936; received by the editors February 23, 1937.

At the time of the reading of the paper no other postulate set for groups or for fields in terms of "indirect" operations was known to me, except Wiener's postulates for fields in terms of "@" (Publications of the Massachusetts Institute of Technology, June, 1920). Since then, there have come to my attention papers on groups treated from my general point of view by $R$. Baer and F. Levi (Zentralblatt, vol. 4, p. 338), Morgan Ward (these Transactions, vol. 32, p. 526), G. Y. Rainich, D. G. Rabinow. Rainich's paper and Rabinow's are, I understand, in press. I have modified the original draft of my paper so as to put in the background possible duplications of the results of these writers. 
$\mathrm{D}_{3}$. If $a, b, b-b$ are in $K$, and if $b \neq b-b$, then $a / b$ is in $K$.

$\mathrm{D}_{4}$. If $a, b, b-b$ are in $K$, and if $b=b-b$, then $a / b$ is not in $K$.

$\mathrm{D}_{5}$. If $a, b$, and the combinations indicated are in $K$, and if $a / b=a / b-a / b$, then $a=a-a$.

$\mathrm{D}_{6} . \quad(a-b) / c=a / c-b / c$,

if $a, b, c$, and the combinations indicated are in $K$.

3. Consistency, necessariness, and independence of Postulates (F). The following arithmetic system $(K,-, /)$ satisfies all the Postulates $(\mathrm{F})$ :

$$
K=0,1 ; a-b=a+b(\bmod 2) ; a / b=a \div b(\bmod 2) \text {. }
$$

Hence, Postulates (F) are consistent.

The properties given by Postulates (F) are seen to be properties of "subtraction" and "division" in a field. Hence, Postulates (F) are necessary for a field.

Finally, Postulates (F) are mutually independent. The independence-systems are given by the table below. The systems in this table are all arithmetic. Some of the systems are modular, the modulus being enclosed in parentheses following the operations. A system contradicting a postulate $P$ is lettered $\bar{P}$. The independence-table follows.

IndePendenCe-Systems For Postulates (F)

\begin{tabular}{|c|c|c|c|c|c|}
\hline System & $K$ & $a-b$ & & $a / b$ & \\
\hline $\begin{array}{l}\bar{N}_{1} \\
\bar{S}_{1} \\
\bar{S}_{2} \\
\bar{S}_{3} \\
\bar{D}_{1} \\
\bar{D}_{2} \\
\bar{D}_{3} \\
\bar{D}_{4} \\
\bar{D}_{5} \\
\bar{D}_{6}\end{array}$ & $\begin{array}{c}\text { Null class } \\
0,1 \\
0,1 \\
0,1 \\
0,1,2,3,4 \\
0,1,2 \\
0,1 \\
0,1 \\
0,1 \\
0,1,2\end{array}$ & $\begin{array}{c}0 \\
a \\
0 \div 0 \\
a+4 b \\
a+2 b \\
a+b \\
a+b \\
a+b \\
a+2 b\end{array}$ & $\begin{array}{l}\text { (5) } \\
\text { (3) } \\
\text { (2) } \\
\text { (2) } \\
\text { (2) } \\
\text { (3) }\end{array}$ & $\begin{array}{c}a \div b \\
0 \div 0 \\
a+b \\
a b+(0 \div b) \\
a+(0 \div b) \\
0 \div 0 \\
a+[0 \div(a b+a+1)] \\
0+(0 \div b) \\
a b+\left(1+2 a^{2}\right)\left(b+2 b^{2}\right) \\
+(0 \div b)\end{array}$ & $\begin{array}{l}\text { (2) } \\
\text { (2) } \\
\text { (5) }\end{array}$ \\
\hline
\end{tabular}

4. Theorems. The theorems $T_{1}-T_{22}$ following are derivable from Postulates $(F)$. They will establish the sufficiency of Postulates $(F)$ for fields.

$\mathrm{T}_{1} . a=b-(b-a)$.

$\mathrm{T}_{2} . a-b=(c-c)-(b-a)$.

$\mathrm{T}_{3} .(a-b)-c=(a-c)-b$.

$\mathrm{T}_{4} . a-a=b-b$.

Definition 1. $0=a-a$. 
Definition 2. $a^{\prime}=0-a$.

DeFINITION 3. $a+b=a-b^{\prime}$.

$\mathrm{T}_{5}$. If $a, b$ are in $K$ then $a+b$ is in $K$.

$\mathrm{T}_{6} . a+b=b+a$.

$\mathrm{T}_{7} . a+(b+c)=(a+b)+c$.

$\mathrm{T}_{8}$. For any two elements $a, b$ in $K$ there is an $x$ in $K$, namely, $x=b-a$, such that $a+x=b$.

In Theorems $T_{9}-T_{16}$ and in Definitions 4, 5, 6 following, unless told otherwise, it is supposed that the elements indicated are all in $K$, i.e. $\left(\mathrm{D}_{3}, \mathrm{D}_{4}\right)$, it is supposed that no "divisor" is 0 . In the proofs of the theorems, "Hypothesis" will refer to this supposition.

$\mathrm{T}_{9}$. If $a=0$ then $a / b=0$, and conversely.

$\mathrm{T}_{10} . a=b /(b / a)$.

$\mathrm{T}_{11} \cdot a / b=(c / c) /(b / a)$.

$\mathrm{T}_{12} .(a / b) / c=(a / c) / b$.

$\mathrm{T}_{13} . a / a=b / b$.

Definition 4. $1=a / a$.

Definition 5. $a_{1}=1 / a$.

Definition 6. $a b=a / b_{1}(b \neq 0) ; a 0=0$.

$\mathrm{T}_{14} .1 \neq 0$.

$\mathrm{T}_{15} . a_{1} \neq 0$.

$\mathrm{T}_{16} .(a / b)_{1}=b / a$.

$\mathrm{T}_{17} .0 a=0$.

$\mathrm{T}_{18}$. If $a, b$ are in $K$ then $a b$ is in $K$.

$\mathrm{T}_{19} . a b=b a$.

$\mathrm{T}_{20} \cdot a(b c)=(a b) c$.

$\mathrm{T}_{21}$. For any two elements $a \neq 0, b \neq 0$, there is an $x$, namely $x=b / a$, such that $a x=b$.

$\mathrm{T}_{22} \cdot a(b+c)=a b+a c$.

5. Proofs of the theorems. The proofs of Theorems $T_{1}-T_{22}$ follow. In $\mathrm{T}_{9}-\mathrm{T}_{16}$, if $a \neq 0, b \neq 0, c \neq 0$, then any combination of $a, b, c$ is in $K$, by $\mathrm{S}_{3}, \mathrm{D}_{3}$. This fact will generally be understood in the proofs.

Proof of $\mathrm{T}_{1} . a=a-(b-b)=b-(b-a)$, by $\mathrm{S}_{1}, \mathrm{~S}_{2}$.

Proof of $\mathrm{T}_{2} . a-b=a-[b-(c-c)]=(c-c)-(b-a)$, by $\mathrm{S}_{1}, \mathrm{~S}_{2}$.

Proof of $\mathrm{T}_{3}$. $(a-b)-c=(c-c)-[c-(a-b)]=(c-c)-[b-(a-c)]$ $=(a-c)-b$, by $\mathrm{T}_{2}, \mathrm{~S}_{2}, \mathrm{~T}_{2}$.

Proof of $\mathrm{T}_{4} . a-a=b-[b-(a-a)]=b-b$, by $\mathrm{T}_{1}, \mathrm{~S}_{1}$. 
Proof of $T_{5}$. By Definition 3, Definition 2, Definition 1, $\mathrm{S}_{3}$.

Proof of $T_{6} . a+b=a-b^{\prime}=a-(0-b)=b-(0-a)=b-a^{\prime}=b+a$, by Definition 3, Definition 2, $\mathrm{S}_{2}$, Definition 2, Definition 3.

Proof of $\mathrm{T}_{7} . a+(b+c)=(b+c)+a=\left(b-c^{\prime}\right)-a^{\prime}=\left(b-a^{\prime}\right)-c^{\prime}=(b+a)+c$ $=(a+b)+c$, by $\mathrm{T}_{6}$, Definition $3, \mathrm{~T}_{3}$, Definition $3, \mathrm{~T}_{6}$.

Proof of $\mathrm{T}_{8} . \quad a+(b-a)=a-(b-a)^{\prime}=a-[0-(b-a)]=a-[(c-c)$ $-(b-a)]=a-(a-b)=b$, by Definition 3, Definition 2, Definition $1, \mathrm{~T}_{2}, \mathrm{~T}_{1}$.

Proof of $\mathrm{T}_{9}$. If $a=0$, then $a / b=(b-b) / b=b / b-b / b=0$, by Definition 1 , $\mathrm{D}_{6}$, Definition 1. If $a / b=0$, then $a / b=a / b-a / b$, by Definition 1 ; hence $a=a-a=0$, by $\mathrm{D}_{5}$, Definition 1 .

Proof of $\mathrm{T}_{10} . \quad a \neq 0, b / a \neq 0$, by Hypothesis. Hence $b \neq 0$, by $\mathrm{T}_{9}$. Hence, $a=a /(b / b)=b /(b / a)$, by $\mathrm{D}_{1}, \mathrm{D}_{2}$.

Proof of $\mathrm{T}_{11} . a \neq 0, b \neq 0, c \neq 0$, by Hypothesis. Hence, $a / b=a /[b /(c / c)]$ $=(c / c) /(b / a)$, by $\mathrm{D}_{1}, \mathrm{D}_{2}$.

Proof of $\mathrm{T}_{12} . \quad b \neq 0, \quad c \neq 0$, by Hypothesis. If $a \neq 0$, then $(a / b) / c$ $=(c / c) /[c /(a / b)]=(c / c) /[b /(a / c)]=(a / c) / b$, by $\mathrm{T}_{11}, \mathrm{D}_{2}, \mathrm{~T}_{11}$. If $a=0$, then $(a / b) / c=0=(a / c) / b$, by $\mathrm{T}_{9}, \mathrm{~T}_{9}$.

Proof of $T_{13} . a \neq 0, b \neq 0$, by Hypothesis. Hence $a / a=b /[b /(a / a)]=b / b$, by $T_{10}, D_{1}$.

Proof of $\mathrm{T}_{14} . a \neq 0$, by Hypothesis. Hence, $1=a / a \neq 0$, by Definition $4, \mathrm{~T}_{9}$.

Proof of $T_{15}$. $a \neq 0,1 \neq 0$, by Hypothesis, $T_{14}$. Hence, $a_{1}=1 / a \neq 0$, by Definition $5, \mathrm{~T}_{9}$.

Proof of $\mathrm{T}_{16} . \quad a \neq 0, \quad b \neq 0, \quad$ by Hypothesis. Hence, $(a / b)_{1}=1 /(a / b)$ $=(b / b) /(a / b)=b / a$, by Definition 5 , Definition $4, \mathrm{~T}_{11}$.

Proof of $\mathrm{T}_{17}$. If $a \neq 0$, then $0 a=0 / a_{1}=0$, by Definition $6, \mathrm{~T}_{9}$. If $a=0$, then $0 a=0$, by Definition 6 .

Proof of $\mathrm{T}_{18}$. If $b \neq 0$, then $b_{1} \neq 0$, by $\mathrm{T}_{15}$; hence, if $b \neq 0$, then $a b=a / b_{1}$ is in $K$, by Definition $6, \mathrm{D}_{3}$. If $b=0$, then $a b$ is in $K$, by Definition 6 .

Proof of $\mathrm{T}_{19}$. If $a \neq 0, b \neq 0$, then $a b=a / b_{1}=a /(1 / b)=b /(1 / a)=b / a_{1}=b a$, by Definition 6, Definition 5, $\mathrm{D}_{2}$, Definition 5, Definition 6. If $a=0$, or $b=0$, then $a b=0=b a$, by $\mathrm{T}_{17}$, Definition 6 .

Proof of $\mathrm{T}_{20}$. If $a \neq 0, b \neq 0, c \neq 0$, then $a(b c)=(b c) a=\left(b / c_{1}\right) / a_{1}=\left(b / a_{1}\right) / c_{1}$ $=(b a) c=(a b) c$, by $\mathrm{T}_{19}$, Definition 6, $\mathrm{T}_{12}$, Definition $6, \mathrm{~T}_{19}$. If $a=0$, or $b=0$, or $c=0$, then $a(b c)=0=(a b) c$, by $\mathrm{T}_{17}$, Definition 6 .

Proof of $\mathrm{T}_{21} . a(b / a)=a /(b / a)_{1}=a /(a / b)=b$, by Definition $6, \mathrm{~T}_{16}, \mathrm{~T}_{10}$.

Proof of $\mathrm{T}_{22}$. We first prove the Lemma $a b^{\prime}=(a b)^{\prime}$. The Lemma is true because, if $a \neq 0$, then $a b^{\prime}=b^{\prime} a=b^{\prime} / a_{1}=(0-b) / a_{1}=0 / a_{1}-b / a_{1}=0-b / a_{1}$ $=\left(b / a_{1}\right)^{\prime}=(b a)^{\prime}=(a b)^{\prime}$, by $\mathrm{T}_{19}$, Definition 6, Definition 2, $\mathrm{D}_{6}, \mathrm{~T}_{9}$, Definition 2 , Definition $6, \mathrm{~T}_{19}$; if $a=0$, then $a b^{\prime}=0=0-0=0-0 b=(0 b)^{\prime}=(a b)^{\prime}$, by $\mathrm{T}_{17}$, Definition 1; $\mathrm{T}_{17}$, Definition 2, hypothesis. If now $a \neq 0$, then $a(b+c)=(b+c) a$ 
$=\left(b-c^{\prime}\right) a=\left(b-c^{\prime}\right) / a_{1}=b / a_{1}-c^{\prime} / a_{1}=b a-c^{\prime} a=a b-a c^{\prime}=a b-(a c)^{\prime}=a b+a c$, by $\mathrm{T}_{19}$, Definition 3 , Definition $6, \mathrm{D}_{6}$, Definition $6, \mathrm{~T}_{19}$, Lemma, Definition 3; if $a=0$, then $a(b+c)=0=0-(0-0)=0-0^{\prime}=0+0=0 b+0 c=a b+a c$, by $\mathrm{T}_{17}$, $\mathrm{S}_{1}$, Definition 2, Definition 3, $\mathrm{T}_{17}$, hypothesis.

6. Sufficiency of Postulates $(F)$ for fields. From propositions $N, T_{5}-T_{8}$, $\mathrm{T}_{18}-\mathrm{T}_{22}$ we see that Postulates (F) are sufficient for a field. ${ }^{*}$

7. Postulates $\left(F^{\prime}\right),\left(F^{\prime \prime}\right)$ for fields. Following are two other sets of postulates for fields:

(F') N, $T_{1}, S_{2}, S_{3}, D_{1}-D_{6}$.

$\left(F^{\prime \prime}\right) \mathrm{N}, \mathrm{T}_{1}, \mathrm{~T}_{3}, \mathrm{~S}_{3}, \mathrm{D}_{1}-\mathrm{D}_{6}$.

In $T_{1}$ is understood the condition if $a, b$, and the combinations indicated are in $K$. In $\mathrm{T}_{3}$ is understood the condition if $a, b, c$, and the combinations indicated are in $K$.

It is clear that the sets $\left(\mathrm{F}^{\prime}\right)$ and $\left(\mathrm{F}^{\prime \prime}\right)$ are each consistent and necessary for a field.

The postulates in each of the sets $\left(\mathrm{F}^{\prime}\right)$ and $\left(\mathrm{F}^{\prime \prime}\right)$ are independent. Independence-systems for $\left(F^{\prime}\right)$ are the same as those for $(F)$, given by the table of $\S 3$, except that systems $\overline{\mathrm{S}}_{1}, \overline{\mathrm{S}}_{2}$ are replaced respectively by $(\alpha),(\beta)$ following:

(a) $K=0,1 ; a-b=0 ; a / b=a+(0 \div b)$.

(B) $K=0,1 ; a-b=b ; a / b=0 \div 0$.

Independence-systems for $\left(\mathrm{F}^{\prime \prime}\right)$ are the same as those for $(\mathrm{F})$, except that systems $\overline{\mathrm{S}}_{1}, \overline{\mathrm{S}}_{2}$ are replaced respectively by $(\gamma),(\delta)$ following:

(r) $K=0,1 ; a-b=a ; a / b=0 \div 0$.

(ס) $K=0,1 ; a-b=b ; a / b=0 \div 0$.

The proof of the sufficiency of $\left(\mathrm{F}^{\prime}\right),\left(\mathrm{F}^{\prime \prime}\right)$ for fields is left to the reader.

8. Postulates for abelian groups. Corresponding to $(\mathrm{F}),\left(\mathrm{F}^{\prime}\right),\left(\mathrm{F}^{\prime \prime}\right)$ we have respectively $(\mathrm{A}),\left(\mathrm{A}^{\prime}\right),\left(\mathrm{A}^{\prime \prime}\right)$ following as postulate sets for (non-trivial) abelian groups:

(A) $\mathrm{N}, \mathrm{S}_{1}, \mathrm{~S}_{2}, \mathrm{~S}_{3}$.

(A') N, $\mathrm{T}_{1}, \mathrm{~S}_{2}, \mathrm{~S}_{3}$.

$\left(A^{\prime \prime}\right) \mathrm{N}, \mathrm{T}_{1}, \mathrm{~T}_{3}, \mathrm{~S}_{3}$.

Clearly, (A), $\left(\mathrm{A}^{\prime}\right),\left(\mathrm{A}^{\prime \prime}\right)$ are each consistent and necessary for abelian groups.

Independence-systems for $(\mathrm{A}),\left(\mathrm{A}^{\prime}\right),\left(\mathrm{A}^{\prime \prime}\right)$ respectively are the independence-systems for the corresponding fields confined to $K$, - .

* Compare E. V. Huntington, Definitions of a field by sets of independent postulates, these Transactions, vol. 4 (1903), p. 33. 
The sufficiency of (A) for abelian groups follows from $\mathrm{N}, \mathrm{T}_{5}-\mathrm{T}_{8}{ }^{*}$ The proof of the sufficiency of $\left(\mathrm{A}^{\prime}\right)$ and of $\left(\mathrm{A}^{\prime \prime}\right)$ is left to the reader. $\dagger$

* Compare E. V. Huntingtcn, Two definitions of an abelian group by sets of independent postulates, these Transactions, vol. 4 (1903), p. 27.

$\dagger$ Since the above was written, two papers by David G. Rabinow have appeared: Independent sets of postulates for abelian groups and fields in terms of the inverse operations, American Journal of Mathematics, vol. 59 (1937), pp. 211-224; Note on the definition of fields by independent postulates in terms of the inverse operations, ibid., pp. 385-392. In the first of these papers the author recapitulates his results in an earlier paper, entitled Independent set of postulates for a group in terms of the inverse operation, offered to the Bulletin of the Society about May 6, 1936. This is the paper of Rabinow's to which I referred in my first footnote. The results in this paper differ little from those in Ward's paper (cited above). As far as I know, the paper has not yet been published. Rabinow's two Journal papers are closely related to mine. (In the first paper, the postulates for abelian groups are precisely my set $\left(A^{\prime \prime}\right)$, without my Postulate $N$ and without the restrictions on the elements in my $T_{1}, T_{3}$.) In neither paper is there reference to Ward's paper or to mine (Abstract 42-5-133, Bulletin of the American Mathematical Society).

The University of California, Berkeley, Calif. 